Article

www.mdpi.com/journal/challenges

\title{
Long-Time Data Storage: Relevant Time Scales
}

\section{Miko C. Elwenspoek ${ }^{1,2}$}

1 Freiburg Institute for Advanced Studies (FRIAS), Albert-Ludwigs-Universität Freiburg, Albertstraße 19, D-79104 Freiburg i.Br, Germany

2 MESA+, University of Twente, P.O.Box 217, 7500 AE Enschede, The Netherlands; E-Mail: m.c.elwenspoek@utwente.nl; Tel.: 0031-53-4893845; Fax: 0031-53 4893343

Received: 8 October 2010 / Accepted: 24 January 2011 / Published: 7 February 2011

\begin{abstract}
Dynamic processes relevant for long-time storage of information about human kind are discussed, ranging from biological and geological processes to the lifecycle of stars and the expansion of the universe. Major results are that life will end ultimately and the remaining time that the earth is habitable for complex life is about half a billion years. A system retrieved within the next million years will be read by beings very closely related to Homo sapiens. During this time the surface of the earth will change making it risky to place a small number of large memory systems on earth; the option to place it on the moon might be more favorable. For much longer timescales both options do not seem feasible because of geological processes on the earth and the flux of small meteorites to the moon.
\end{abstract}

Keywords: Human Document Project; data storage; memories; long-term processes

\section{Introduction}

All branches of natural sciences report one common result: our world is continuously changing. We live in a dynamic world and structures that seemed to be fixed are actually evolving; however on a time scale much longer than human life or the human memory.

Natural sciences uncovered many of the rules that govern this change. Some processes are rather simple and well understood, such as the lifecycle of a star of the mass of our sun. Others are very complicated, such as the varying orientation of the magnetic poles on earth, which are not well understood.

The rules themselves do not seem to change, however. Cosmologists make observations and theories on the development on the very grandest scales of the universe, and on the longest time intervals. This is possible because the speed of light is finite, therefore, when astronomers aim their 
instruments at distant objects they also look into the past. What can be seen suggests that the rules have been the same since the birth of our universe, 13.7 billion years ago, see for example, [1].

The memory of our personal lives goes back a little beyond our actual lifespan: we heard stories told by our parents and grandparents, only occasionally has someone the luck of great-grand parents. Beyond theses generations the past becomes very abstract. We oversee only a small fraction of the roughly 200,000 years Homo sapiens sapiens existed. The oldest written script seems to be Cuneiform script developed by the Sumerian culture [2] which dates back to the 23 rd century before our time. The Gilgamensh epos is a prominent example of very early texts. From this epos we get an impression about the ideas of the people of their time; hence we oversee only a little more than 4,000 years of history of human thinking - this is a mere $2 \%$ of the existence of Homo sapiens sapiens.

Notwithstanding these observations, we have a keen interest in our history. New discoveries of ancient artifacts make headlines in the papers, and the discovery of a civilization under Antarctica's ice, no matter how old, would be a sensation, even more so if it were not of human origin. This fact constitutes a strong argument in support of storing data on human kind for a very long time.

Modern data storage media faithfully conserve information for not much more than a mere 10 years. The VCR tapes showing my children are badly corrupted. CDs, even when kept in a safe environment do not last much longer. Special paper may survive 1,000 years, see for example, [3]. Script carved in stone may survive a very long time, maybe one million years if it is protected from erosion. But the amount of information that can be stored by writing on stone is small. It would be impossible to encode a movie on stone.

Actually, securely storing large amounts of information over quite short timescales, comparable to our personal memory span of say 100 years, is not trivial at all. Even for timescales of 10 years there is a big problem; the amount of data we are collectively creating is enormous. Attempts to conserve all data face the problem that the time required to copy the data is longer than the time in which data storage systems change, so much so that there is no simple way to transfer the data to the new system [4]. Think of magnetic tapes, floppy discs, CD's, DVD's, Blue ray etc. To the author's knowledge, there is no product on the market that provides a high-density medium, for failsafe data storage for 50 years, which is compatible to modern information technology. So, even moderate timescales pose a technological problem.

In this paper processes of relevance for high-density data storage over very long timescales are reviewed. These processes are due to the expansion of the universe, astrophysics which describes the formation and evolution of stars and dynamic processes within our galaxy, the local cluster of galaxies and the solar system, geological processes on earth such as erosion, mountain formation, plate tectonics, volcanism and atmospheric processes, and finally biological evolution.

A target timescale for the human document project is of high importance: the timescale defines the properties of the media and the system and will tell us something about the potential readers - human or non-human, and on the possible locations where the system can be stored.

It is found that one million years is a sensible aim. However, it is problematic to store a very large amount of data on earth. Such a system might be implemented on the moon. It might make sense to fabricate many copies of small amounts of information and distribute them over the earth leading to a high probability that a few will be found in a million years time containing retrievable data. 


\section{Cosmology}

We live in an ever-expanding universe; for a recent review see [5]. One of the most popular descriptions of the birth of the universe is in [6]. The expansion means that in earlier times the galaxies were closer together. There must have been a time when there were no stars, no galaxies. The temperature of the universe must have been higher the farther back in time we go, and before a certain time, called the "recombination time", the universe must have been so hot, it was filled with a plasma in which light could not propagate freely. The moment the plasma (hydrogen and helium nuclei and free electrons) became neutral by forming atoms of hydrogen and helium (in atoms the electrons are bound), the existing light could propagate. We still can see this light as the "cosmic microwave background" (CMB). The cosmic expansion stretched the wavelength of the CMB from the visible range to microwaves by now. The microwave background is nearly isotropic, which means that the properties of this light are nearly the same regardless of which direction it is viewed. The temperature of this light (average $2.7 \mathrm{~K}$, where $\mathrm{K}$ is the scale of absolute temperature, $0{ }^{\circ} \mathrm{C}=273 \mathrm{~K}$ ) deviates from the mean by only 1 in 100,000 . This deviation led to the formation of galaxies. The anisotropy of the $\mathrm{CMB}$ gives us clues to a number of basic properties of our cosmos: Its age, its geometry, its mass content and many other things [7]. Very much to the surprise of cosmologists, only four percent of all matter in the universe is ordinary matter, of which we, planets and stars are made. The rest is so-called dark matter, consisting of some yet undetected elementary particles that do not interact with light, and-even most of it - so-called dark energy. No one knows what the latter is, but we do know that this is responsible for the observed accelerated expansion of the universe.

First, stars were formed many hundred million years after the birth of the universe. The oldest galaxies we see with our telescopes were formed within a billion years. These ancient stars and galaxies were quite different from the modern stars and galaxies. This is because there was no other material than hydrogen and helium. Heavier materials such as oxygen, nitrogen and carbon were formed in the cores of stars and redistributed in the galaxies by exploding stars. It took billions of years before enough heavy materials were synthesized in stars so that rocky planets were formed. Life as we know it is dependent on stars which deliver energy needed for metabolism. It also seems to require surfaces of rocky planets and a stable planetary system and an environment free of intense radioactive radiation. These conditions restrict regions where life is possible to a certain part of our galaxy: not too close to the nucleus because of the radiation level and the greater chance of supernovae, and not too far because of the need of heavy elements. In this region of our galaxy sufficient material to form rocky planets has existed for about six million years; there is sufficient material to form rocky planets in the centre of the galaxies, but the intense radiation background cannot tolerate complex organic molecules [8].

Star formation will stop eventually, when the hydrogen gas becomes too diluted. The last stars will die in about $10^{14}$ years (100 zillion years) [9]. Observational results rule out a collapsing universe: it will expand forever.

There is a large body of literature in which possibilities are analyzed to continue processes analogous to thought when there are no stars left. In an abstract way thought is some type of data manipulation. Freeman Dyson's paper [10] was the first dealing with an analysis of possibilities for eternal life in an expanding universe. A more recent account on this subject can be found in 
reference [11]. With the knowledge we currently have, we must conclude that life will end. The basic reason is that eternal life would need an infinite amount of energy, but the evolution of the cosmos gives access only to a finite volume of space, containing a finite amount of energy. Since there is strong evidence for an accelerating expansion of the universe [12], we have to adopt the worst scenario possible in an ever-expanding universe.

Galaxies interact. In particular, the giant galaxy Andromeda will merge with our galaxy [13]. Given the uncertainty of a number of parameters, it is not known what exactly will happen to us, nor when. Certainly the merger will happen within the lifetime of our sun; possibly it will start within the next billion years. There will be no head-on collision between stars because the distance between stars is too great (the ratio of the size of a star to their average distance in a galaxy is comparable to that of a rowing boat to the size of the Pacific Ocean; the chance that two rowing boats collide incidentally is very small). But the stars will be mixed up; a star could come close enough to our solar system to disturb the planets' orbits. The greatest danger is expected to result from the increased rate of star formation induced by the merging galaxies. More new stars mean many new massive stars with a short lifetime. There will be many more supernova explosions. Furthermore the orbits of stars will be such that there is a big chance that our sun comes close to active star forming regions, or even to the centre of one of the galaxies. Here conditions for life are quite unfavorable due to the high radiation level. Such an adventurous trajectory through the merging galaxies might not necessarily end all life, but very probably complex life such as animals and plants. Bacteria might survive this period.

Figure 1. Schematic of the sun's luminosity.

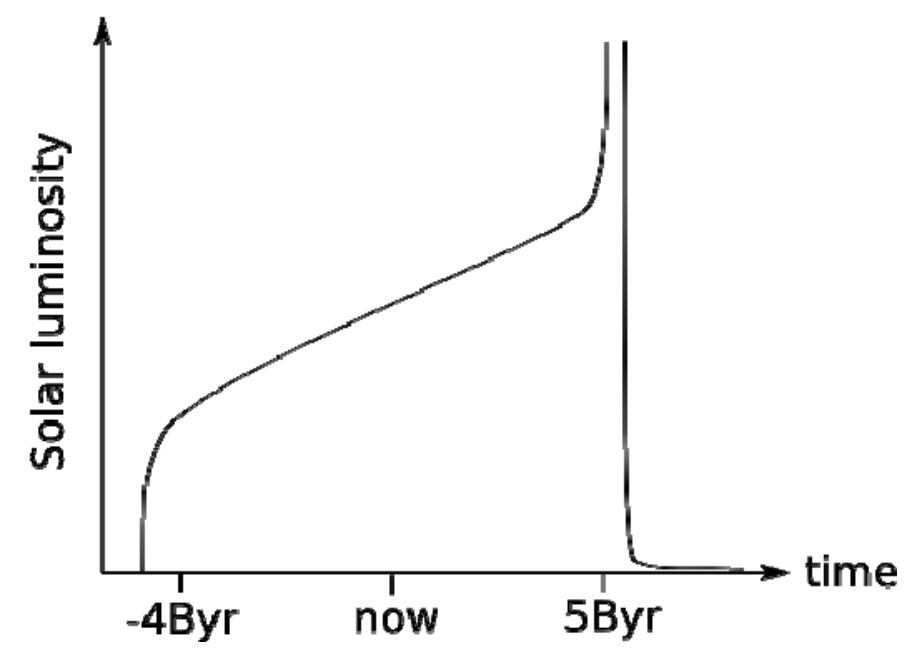

\section{Astrophysics}

The life cycle of our sun is shown in Figure 1. The sun will remain a star as we know it for the next five billion years. Since its formation its temperature has increased. When life started on earth about 3.5-3.8 billion years ago [14], the radiation intensity was 30\% less than today. The sun's temperature will continue to rise. In a billion years no liquid water will exist on earth. A runaway greenhouse gas effect might cause an earlier boiling away of the oceans. The total window for the existence of complex life, such as mammals might be not wider than a billion years; half of it might have already been used up [15]. In later stages, after five billion years, the sun will swell into a red giant, the earth's 
orbit could be inside the star, then the temperature of the earth will be 3,000 K; no manmade structures will survive on the earth or the moon.

In this view, it would make no sense to create a system located on earth or the moon which has the capacity to store information longer than a billion years. It might be feasible to send a robotic space ship into the galaxy and let it search for a cooler star with planets, land on one of them, and use the star's radiation to build a beacon sending information of our existence into the galaxy. Cooler stars have a much longer lifetime than stars of the sun's type [16]. So-called m-stars may shine for nearly a 100 billion years.

The increasing intensity of the sun's radiation will have quite unpleasant consequences for complex life on earth. Although the solar intensity was smaller in the past, the earth was warmer in general. The earth's temperature remains roughly constant due to a geological feedback loop; an early famous paper on this matter is [17], a modern account can be found in [18]. Higher temperature leads to more erosion, which removes $\mathrm{CO}_{2}$ from the atmosphere faster. The cooler sun was offset by a higher concentration of greenhouse gas. In the course of time the $\mathrm{CO}_{2}$ level decreased due to the increased energy influx from the sun. Decreasing $\mathrm{CO}_{2}$ levels causes the temperature of the atmosphere to drop. The concentration of greenhouse gas will continue to decrease. The current ice age is possibly a consequence of low $\mathrm{CO}_{2}$ concentration. Ultimately the deceasing $\mathrm{CO}_{2}$ will have consequences on plant growth. Plants use $\mathrm{CO}_{2}$ in photosynthesis by which $\mathrm{CO}_{2}$ is removed from the atmosphere and used to synthesize the organic material which the plants are made of. Oxygen is released into the atmosphere in this process (this is the reason for the existence of free oxygen in the earth's atmosphere). Below a certain concentration of $\mathrm{CO}_{2}$ photosynthesis ceases; for grass-like plants the critical concentration is about $10 \mathrm{ppm}$ while other plants need an even higher $\mathrm{CO}_{2}$ concentration. Model calculations predict than in 500 million years, plants will have vanished, and a little later animals will perish, too [19]. Only bacteria will continue to thrive, once again becoming the master of the world, as they were for the first three billion years of life on Earth. The window in which complex life is possible is bracketed between a minimum oxygen concentration (roughly at the Cambrian Explosion 530 million years ago) and a minimum $\mathrm{CO}_{2}$ concentration to enable plant growth; this window has a duration of about a billion years. We are half way through this. On other earthlike planets orbiting suns like ours there will be a similar window.

Increasing temperature on earth will lead to a higher concentration of water vapor in the atmosphere. $\mathrm{H}_{2} \mathrm{O}$ is also a greenhouse gas, so the temperature will rise, leading to increasing evaporation of the oceans, so again the temperature will rise, and so on: this positive feedback loop leads to a runaway greenhouse gas effect, possibly causing surface temperatures as high as on Venus $\left(400{ }^{\circ} \mathrm{C}\right)$. Water molecules will be split up by the sun's ultraviolet radiation. Then hydrogen will disappear into space. This process might change the scenario to a less grim one if it is fast enough. Of course, there will be no one around able to benefit from this except for bacteria.

\section{Geology}

The Earth is a very active planet. A relevant consequence for the Human Document Project is that the surface of the earth changes in the course of time. Earth's surface is made up of continental plates that move with a velocity relative to each other which is in the order of $2 \mathrm{~cm} /$ year. Parts of the 
continents move up, others move down causing shifts in coast lines. About 250 million years ago nearly all landmass was connected to a single large continent. This will happen again in 250 million years [19]. All high mountains we now have on earth started to form about 50 million years ago.

Also erosion and redeposition of eroded material changes the surface. High mountains will be gone within 50 million years. Erosion rates differ greatly, dependent on the location. Within a few million years new lakes form, and rivers change their course. Caves have a lifetime of perhaps 10 million years. Landscapes change beyond recognition within a few million years. In the "Stadtspark" of the city of Freiburg, Germany, there is a plaque situated right on top of a fault line marking the border between the Black Forest and the valley of the river Rhine. This plaque shows a map of Germany as it was 30 million years ago. Had Freiburg been built then it would lie at the sea shore.

During the past 2.5 million years, $90 \%$ of the time parts of the northern hemisphere and Antarctica were covered by glaciers. We have since been living in an ice age. Some glaciations were so severe that a large proportion of the U.S. and middle Europe was under a kilometre thick layer of ice. The current time period is a so-called "interglacial", a warmer period with relatively little ice. Interglacials occur about every 100,000 years. Warm periods usually did not last much longer than 10,000 years. The last glaciation ended about 12,000 years ago, meaning the next one must be expected soon, but perhaps global warming causes a delay of a few hundred years. The next glaciation period will have drastic effects on our culture, probably much more severe than global warming. While global warming will lead to a rise in sea level, perhaps by $6 \mathrm{~m}$ or so, leading to massive loss of coastal areas, a major glaciation period will lead to a decrease in sea level because lots of water will be locked up in ice. Loss of habitable land in the moderate north will have a greater effect, however, on our culture. Probably more importantly, the climate will be much drier, so where there is no ice on the northern hemisphere there will be savannas and deserts. With current technology it will be impossible to feed today's population.

Within the next million years this will happen about 10 times. Possibly, mankind will survive this, but our culture will be under great stress most of the time. Therefore there is a good chance that our culture will vanish and all our accomplishments in science and humanities will disappear. It is even not so improbable that man will disappear altogether.

Without a shadow of doubt, after a million years our traces will be found everywhere on earth, certainly even after a few 100 million years many signs of our culture will still be obvious. However, a system placed on some selected location will probably be destroyed. It will be quite impossible to predict what specific trace will remain over such long time intervals. This has two consequences: It makes little sense to direct the Human Document Project only to notify later civilizations of our existence. This is not necessary in view of the many traces we shall leave. Second a small number of systems carrying large amounts of information placed somewhere on earth will be useless: the probability that the systems will never be found or that they will be destroyed is too great, even within a million years.

\section{Global Catastrophes}

From geological and paleontological records, we know that earth suffered a number of extinction events; since animals and plants on land have existed, there were five major catastrophes during which 
a large proportion of species vanished. The disappearance of species occurred in a short time scale in terms of geological time. Figure 2 shows the extinction rate since the Cambrium. Of the five major events, the most prominent event was at the end of the Perm, about 252 million years ago.

There are several mechanisms known that may lead to relatively sudden mass extinction. These are climate change, volcanic eruption, meteorite impact and nearby supernovae.

Figure 2. Extinction rate since the Cambrium. Note the apparent periodicity; it stirred up a lot of attention in the 1980s [20], however, up to now a systematic periodic cause of extinction events has not been confirmed. Data from [21].

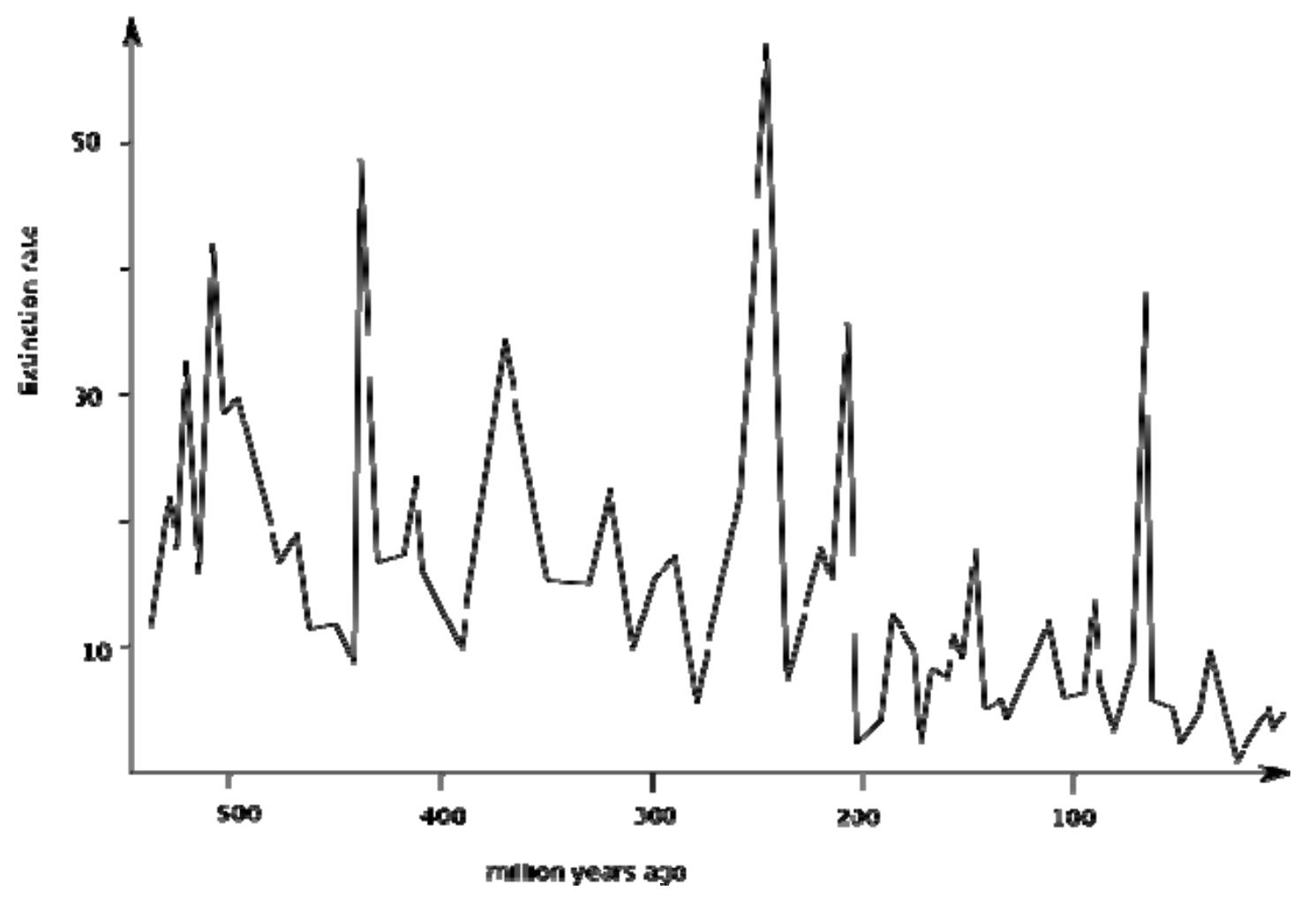

To begin with the last of these, there are indications that in the very early history of the planet a supernova occurred close enough to earth [22] which would have caused massive damage to life if it was formed by then. The sun was formed in an open cluster [22] together with a large number of other stars. Today we can see similar processes in the galaxy; most well known may be the star formation region in the Orion nebula [23]. Typically stars of a wide range of masses are formed. The larger the mass the shorter the star lives, and the end of massive stars is in the form of a super nova if its mass is large enough (in the order of 10 times the mass of our sun). This cluster may have contained as many as 3,500 stars. By now it has dispersed due to tidal forces caused by the galaxy.

Evidence exists for nearby supernovae in the (geologically) recent past [24]. One, the radiation of which reached us two million years ago, might have triggered an extinction event in marine life by damaging the protecting ozone layer. In general, we might state that the chance of a supernova explosion close enough to earth to extinguish life on a very large scale is rather small. The earth, on its way through the galaxy is unlikely to encounter an active star-forming region. Where there are older stars, no supernovae occur because only very massive, and therefore short lived, stars can turn into a 
supernova. We discussed, in Section 2, the predicted collision of the Andromeda galaxy with ours; this process will change the situation.

So-called x-ray bursts are also the result of supernova explosions. The radiation emitted by an exploding star is not isotropic; instead most energy is concentrated in a narrow jet emerging on either pole of the star. In the case that earth was hit by such a jet, the radiation would be dangerous for life also if the star was much further away. The star which exploded two million years ago was at the time of explosion at a distance of 300 light years from us. To be disastrous to life on earth it would need to be considerably closer than one light year. However, being hit by a jet a few hundred light years distant would pose a serious threat. Fortunately, the powerful jets are very narrow. The only supernova that exploded close enough to the earth to have had the potential of causing direct massive extinction by its radiation, was the one shortly after the solar system was formed. It did no harm to life just because there was nothing alive at the time.

Contrary to that, meteorite impact on earth is occurring much more frequently. The activity of the earth conceals most of the impact craters. Still, the earth impact data base [25] lists nearly two hundred impact craters, ranging in size from $150 \mathrm{~m}$ to $300 \mathrm{~km}$. Since our moon has little or no geological activity (certainly no tectonics) the traces of many more impacts can be seen. Wikipedia lists about 1,200 craters, with diameters from $2 \mathrm{~km}$ up to $2,240 \mathrm{~km}$. So we must conclude that Earth has been hit by meteorites many times, perhaps even more frequently and heavier than the moon because of its greater gravitational field. The first half billion years of the earth's existence was characterized by a heavy bombardment, one of the impacts is believed to have led to the formation of our large moon [26]. Many of the impacts were such that the whole surface of the earth melted. The flux of large objects towards earth steeply decreased about four billion years ago, when most debris left over from the formation of the solar system was cleared. Still, now and then big comets or asteroids hit the earth. One of them, of about ten kilometers in diameter, fell on the Yucatan peninsula to form the Chicxulub crater [27], now Mexico, and caused a global catastrophe at the boarder of the Cretaceous and Tertiary $(\mathrm{C} / \mathrm{T})$. Its most well known effect was the extinction of the dinosaurs, but many other families in the oceans and on land became extinct.

The relationship of other great impacts, for example, the possibly multiple impact in the late Triassic in Canada and Europe [28], with global mass extinction, is less clear. In this case the meteorites had masses about ten times smaller, therefore the consequences were much less than that of the $\mathrm{C} / \mathrm{T}$ event. The relation of the size of the impacting body with the damage it causes is shown roughly in Figure 3. Note that besides the size, also the composition and the impact site plays a role. A meteorite falling into the ocean would make a wave of a height either similar to its diameter or to the depth of the sea, whichever is smaller. The $\mathrm{C} / \mathrm{T}$ asteroid would have caused a wave with an initial height of $4000 \mathrm{~m}$ had it fallen into deep ocean. 
Figure 3. The relation of the size of a meteorite to the damage it would cause. Data from [29].

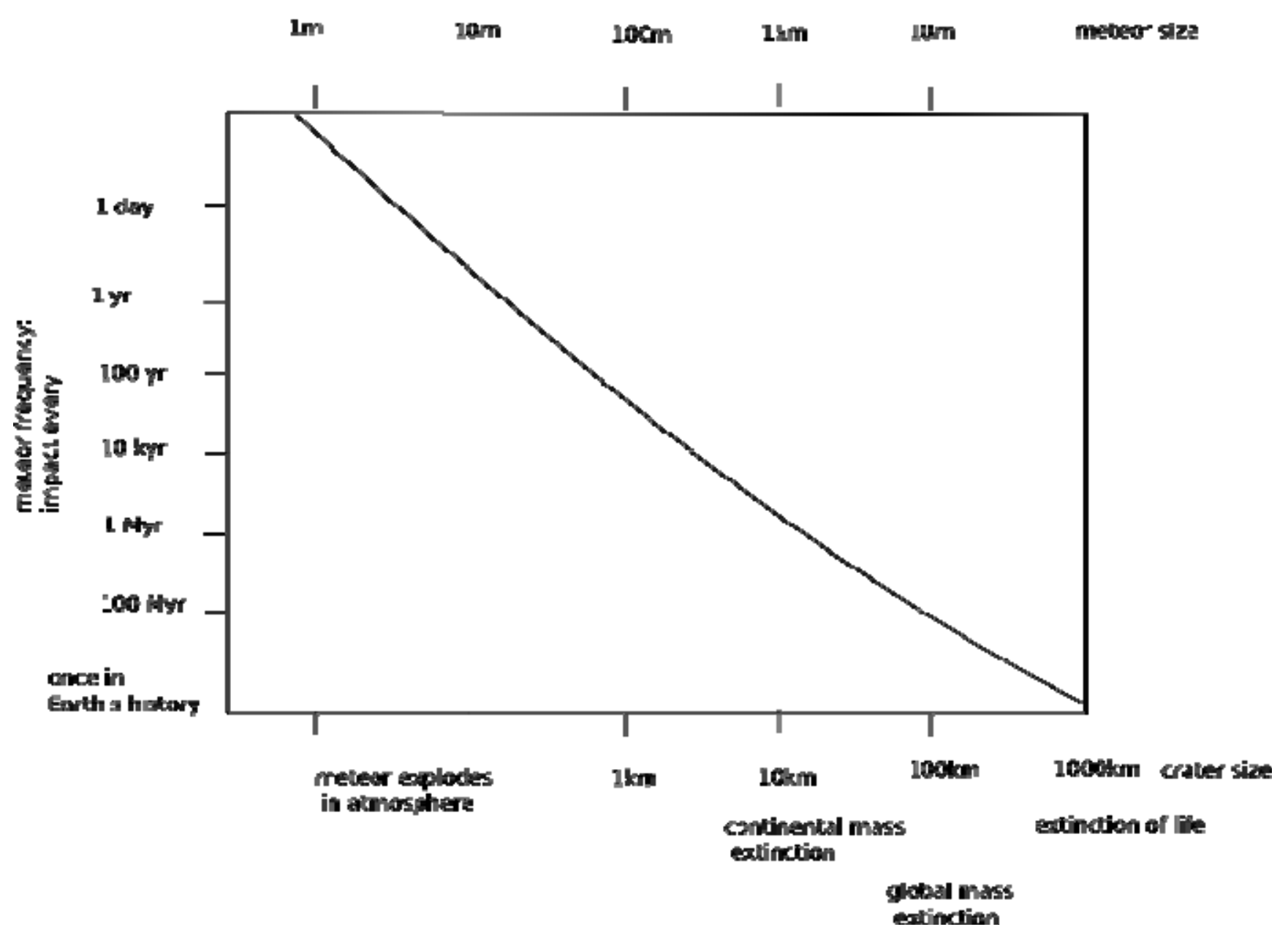

The current view is that it was the geology of our planet that was responsible for most of the mass extinctions. An example is the greatest known extinction event ever, at the transition from the Perm to the Triassic $(\mathrm{P} / \mathrm{T})$, which is described in a wonderful book by Michael Benton [30]. At this time all continents formed a single super-continent known as Pangea. Similarly to other great extinction events, with the exception of the one caused by the $\mathrm{C} / \mathrm{T}$ meteorite impact, life forms did not vanish suddenly but the dying occurred over many hundreds of thousands of years. During this period there were rapid extinction events interspersed that came in several bursts and the time taken for the ecosystem to recover was substantially longer. The severity of the P/T event is attributed by Peter Ward mainly to the existence of the Pangea supercontinent [19]. The trigger almost certainly was a volcanic eruption of a quite unimaginable magnitude. In Siberia there is a geological formation called Large Igneous Provinces, or Siberian Traps so named because of their staircase-like appearance. The traps consist of a few million $\mathrm{km}^{3}$ of volcanic rock. Originally, an area larger than Europe was covered by the lava; now some of the rock has eroded away. The eruption lasted a few hundred thousand years, perhaps even a million years [31].

There are many similar volcanic deposits of enormous size on the planet, but the Siberian traps seem to be the largest. Currently a Large Igneous Province is being formed in the Iceland area. 
Somewhat smaller volcanic systems are the so-called super volcanoes (this term was apparently coined by a BBC program on these features; there was an impressive movie aired by BBC in 2005 under the title Supervolcano, describing the possible consequences for the U.S. if Yellowstone park explodes). Table 1 gives an overview of modern super volcanoes (the Siberian Traps are included to illustrate the difference of scale between super volcanoes and those volcanic systems that formed the traps). The eruption at Lake Toba, 74,000 years ago is possibly responsible for a period during which the population of Homo sapiens seems to have been at a minimum; this has been concluded from genetic evidence [32,33]; however, the hypothesis is under debate [34].

The hot spot lurking under the Yellowstone Park would, if it exploded, cause major problems to North America. The last time this super volcano went off, the western part of U.S. was covered in a layer of ash. The eruption would cause millions of immediate casualties, disrupt the infrastructure of Canada and the U.S., destroy the possibility for agriculture in the prairies for years and cause a series of cold summers resulting in poor harvests and severe famines worldwide. It would not extinguish humankind, however. The odds are high that it will explode during the next few thousand years, and that within the next million years it will certainly erupt several times, as will the other super volcanoes listed in Table 1.

Table 1. Super volcano eruptions.

\begin{tabular}{|c|l|c|}
\hline $\begin{array}{c}\text { Date } \\
(\mathbf{1 , 0 0 0} \mathbf{~ y r s})\end{array}$ & Location & $\begin{array}{c}\text { Material ejected } \\
\left.\mathbf{( 1 , 0 0 0} \mathbf{~ k m}^{\mathbf{3}}\right)\end{array}$ \\
\hline 25.5 & Lake Taupo, North Island, New Zealand & 1.2 \\
\hline 74 & Lake Toba, Sumatra & 2.8 \\
\hline 254 & Lake Taupo, North Island, New Zealand & $1.2-2$ \\
\hline 640 & Yellowstone, US & 1 \\
\hline 2,100 & Island Park, Idaho/Wyoming, US & 2.5 \\
\hline 2,500 & Cerro Gala, Argentina & 1.1 \\
\hline 4,000 & Atana Ignimbrite, Chili & 2.5 \\
\hline 4,500 & Yellowstone & 1.5 \\
\hline 29,500 & Sam Ignimbrite, Yemen & $>5.6$ \\
\hline 250,000 & Siberian Traps & $1,000-4,000$ \\
\hline
\end{tabular}

Many extinction events can be correlated with major volcanic activity [19]. The exact mechanism of how the eruptions may cause the massive extinction is not as yet clear. Besides ash and lava volcanoes emit gases, notably $\mathrm{SO}_{2}$ and the greenhouse gasses $\mathrm{CO}_{2}$ and methane. Ash and $\mathrm{SO}_{2}$ would contribute to immediate and short-term climate change by reducing the amount of sunlight that reaches the earth's surface, causing a temperature drop. These effects however have a short duration: within a year or so the atmosphere is cleared provided the eruption stops. The enormous amount of gasses may lead to an effective reduction of the oxygen content in the atmosphere and the oceans. The greenhouse gases have a long lasting effect, reversing the decline in temperature in the long run.

Peter Ward describes the following grim scenario for the P/T event [35]: The higher temperature of the oceans reduces the solubility of oxygen in water. A stagnant ocean, i.e., an ocean without currents mixing solvent concentration would have lost most of its oxygen, enabling a special type of bacteria to thrive. These bacteria do not use oxygen in their metabolism but sulfur; their waste product is $\mathrm{H}_{2} \mathrm{~S}$, 
which accumulates in the oceans. A stratified sea would result, similar to what is seen in the Black Sea. Bacteria that use sunlight and produce $\mathrm{H}_{2} \mathrm{~S}$ proliferate if the surface of the oceans is free of oxygen, producing even more $\mathrm{H}_{2} \mathrm{~S}$. This gas, being lethal to plants and animals alike, can be released in gigantic eruptions into the atmosphere. While most higher lifeforms in the oceans were killed due to the lack of oxygen and poisonous $\mathrm{H}_{2} \mathrm{~S}$, life on land perished, too.

\section{Placement on the Moon}

It can be concluded that the earth might not be a good choice for the placement of a large long-term memory system; however the moon could be, because it is geologically inactive. Threats to the moon come in the form of meteorites which constantly strike its surface, being unprotected by an atmosphere.

Impacts of microscopic particles lead to some erosion of the moon's surface. This erosion rate however is much smaller than erosion rates on earth; the system would be well protected from small impacts by burying it a few meters under the surface.

Impacts of larger bodies are destructive. The flux of large bodies to the moon is quite small, and more importantly, the damage caused to the moon's surface is local because of the lack of an atmosphere. In order to destroy the system a meteorite would need to impinge the surface at a distance from the system closer than about 30-50 times the diameter of the impactor. Only the resulting moonquake could pose damage to the system when the impact is further away. This should not be a problem because the system will not require mechanically delicate parts.

The frequency of impacts of large bodies of $1 \mathrm{~km}$ diameter or more has been given by Mathieu Le Feuvre [36] to be close to $10^{-8}$ impacts per year. $50 \mathrm{~m}$ sized objects impinge on the earth only every 10,000 years; in the range of $5-10 \mathrm{~m}$ the rate is one per day [37]. These objects do not reach the surface of the earth because they evaporate due to friction in the earth's atmosphere. They strike the moon surface, however, with a similar frequency [38], reduced by the ratio of the surfaces of the earth and the moon, which is about 16 . A hit of a $5 \mathrm{~m}$ object at a distance closer than 200-300 $\mathrm{m}$ from a data storage system would be destructive [39]. The chance that such an object would strike within a distance of one hundred meters within one million years would be in the order of $2 \%$ (i.e., number of impacts in one million years times $200 \times 200 \mathrm{~m}^{2}$ divided by 16 , divided by the surface of the moon: $3.6 \times 10^{8} \times 0.04 \mathrm{~km}^{2} / 16 \times 4 \times \mathrm{p} \times 1,700^{2} \mathrm{~km}^{2}$ ), dangerously close to one in view of the rough assumptions we made.

\section{Evolution}

The pertinent questions are: What changes have to be expected regarding the biosphere? Who will attempt to understand the human document? Will it be people like us or a new sort of homo or even a species derived from a different family?

The statistics of evolution and lifetimes of a family or species are quite complex (family refers to cats; a species would be the lion). It has been estimated that on average 0.5 to 5 species become extinct every year [40] and among mammals the extinction rate is about one species per year [41]. The mass extinctions which have been referred to in Section 5 are at the top of this background extinction rate. It is not well known how many species there are, but in [42] about 5,000 species of mammals are 
described. A background extinction rate of one species per year would mean that the average lifetime of a mammal species was only 5,000 years or between 1,000 and 10,000 generations, which seems to be very short. This figure is misleading because the statistics are not simple; in the distribution of the lifetime of species there is an exponential decay, which might have a characteristic time of thousands of years, but there is a long tail. This indicates that there are many species that vanish quickly, but other species survive much longer, some by orders of magnitude. The geological record gives a picture of the extinction dynamics of species [43] showing this trend, see Figure 4 for the lifetime distribution. It can be seen that most families have a lifetime of 10 to 20 million years, but there are some with lifetimes of many hundreds of millions of years.

These statistics make it difficult to predict a reasonable lifetime for a specific species, in particular that of Homo sapiens. It might be possible to argue that intelligence would lead to a particularly long lifetime, but the contrary to that could well be put forward. So intelligence might have no influence on the chance of survival in the longterm.

Looking back, we see that the homo family separated from the ancestor of today's chimpanzees about six million years ago [44]. Since then, more than 10 species of homo are known from the quite incomplete paleontological record. No one knows whether this early homo had a particularly good chance to develop an intelligence that would allow him to develop a language and symbolic thought. Therefore nothing sensible can be said on the chance of, say, a species of rodents turning into one with a similar capability. It seems to be impossible for insects, though, since they have existed for several hundred million years and have never developed intelligence. Dolphins might have the capacity to develop higher intelligence, but it is difficult to see how they would experience pressure to develop something equivalent to written language. Therefore it might be stated that a species, outside the homo family, capable of symbolic thought and therefore capable of understanding any human document will take many millions of years to evolve from the present state.

Homo sapiens are still evolving [45]. The present globalization is such that races are constantly mixed. As long as the gene flow within humanity is of such a magnitude, there is no chance of a split of Homo sapiens into two or more species. Catastrophes, such as ice ages or super volcano explosions, that surely will come, might disrupt our culture and separate groups of people for a long time. However it might be doubted whether this time is long enough to form species unable to interbreed. Neanderthal man, so very recent results of their genes tell us, interbred with us - they did not become extinct but mixed with us and simply left their characteristic sites [46]. The Neanderthals left Africa a few hundred thousand years earlier than "we" (as "we" were formerly thought to be a species separate from the Neanderthals; the we is no longer appropriate), and still after such a long time we and the Neanderthals did interbreed. Therefore it is quite unlikely that, even after a 100,000 year long cold and dry period of heavy glaciation, separate settlements of Homo sapiens would not find each other in a warmer interglacial environment and interbreed. Evolution will go on but is unlikely to result in a new homo species within the next million years.

This section is necessarily quite speculative; therefore we refrain from making any guesses over longer times. Whoever finds the human document in a hundred million years, will most probably not be human. 


\section{Discussion}

A static data storage system is defined here as a structure, into which the information is coded in some way and left alone. An active data storage system would monitor its own state and repair any damage. While the latter needs energy, the former does not. A static information system inevitably deteriorates due to thermal fluctuation. The temperature in the universe will be finite always, and so will the fluctuations. In principle, the active system could last much longer than the static one, as long as it is capable of mining energy.

From the presently available data on our cosmos it must be concluded that life will not exist indefinitely. Since an active data storage system requires energy, its lifetime will be finite-this follows from the same arguments about why life cannot exist forever. Any document stored for later retrieval therefore must be designed for a finite lifetime. What might be a sensible lifetime to aim for in the design?

An artificial active, autonomous data storage system is way beyond today's technological capabilities (a system which is not autonomous, i.e. which needs human aid, makes no sense). Such a system would rightly be called alive. Therefore presently all we can think of is a static data storage system.

Any data storage is in some way or other related to a structure. This means there are regions in space containing some material of another type or with a different property such as magnetization, than its surroundings. In principle such a system is outside thermal equilibrium. It is wise to use a medium which is chemically not reactive and, left alone, is in or close to thermal equilibrium with its surroundings. For instance, the most reactive component in our atmosphere is oxygen, so the material should be an oxide such as quartz or sapphire. Data could then be stored by having sapphire dots in a quartz matrix. The slowest process of deterioration in such a system probably is a diffusion process. This sets the maximum possible lifetime of the storage system: it is the diffusion time of atoms and molecules between the structures. This time $\tau$ is given by

$$
\tau=\frac{\lambda^{2}}{D}
$$

here, $\lambda$ is the distance between the information carrying structures (e.g., the size of a letter or the distance between the dots in a DV). $D$ is the diffusion coefficient.

In solids, the diffusion coefficient at room temperature is usually immeasurably small. We might estimate a lower limit for the diffusion coefficient by noticing that ancient stones and meteorites seem to have preserved much of their microstructure, meaning that the diffusion length in metals and silicates is below $1 \mathrm{~mm}$ in a few billion years. The corresponding diffusion coefficient is then in the order of $10^{-30} \mathrm{~m}^{2} / \mathrm{s}$. So one has to extrapolate from measurements at high temperature which usually leads to much smaller diffusion coefficients [47] (in this paper an activation energy of $3.4 \mathrm{eV}$ is given, which translates into a diffusion constant at room temperature of $10^{-70} \mathrm{~m}^{2} / \mathrm{s}$, leading to a diffusion length of $10^{-25} \mathrm{~m}$ since the existence of the universe).

State of the art data storage systems use dot distances of about $100 \mathrm{~nm}$ or less. Equation (1) shows that the greater the data density, or the smaller the system, given a fixed quantity of information to store, the faster the systems "forgets" its information. Generally, the diffusion coefficient increases 
steeply with temperature, therefore it is wise to keep the system at the lowest temperature possible. It seems that diffusion is slow enough to permit a static data storage system preserving information over many billions of years. Therefore the bottleneck is the protection of the system against thermal, chemical and mechanical stress.

We have seen that it does not make sense to design a data storage system intended to be found and read by earthlings lasting longer than about 500 million years. This might be technologically feasible.

A more durable system might make sense if it is put on a spacecraft to be found by alien civilizations outside the solar system (or by our descendents, in case interstellar space travel is possible). However, the density of stars is so low that the chance that it will ever be found by coincidence, for example, when it has a close encounter with a star orbited by a life-carrying planet, is vanishingly small. Such a system would require a robot to send signals into the galaxy now and then indicating its position whenever it is reasonably close to a star, in any case close enough to mine energy from the star. It is doubtful whether this is feasible with current technology.

It might be possible to develop technologies that allow us to colonize the galaxy. But the technological challenges for interstellar travel carrying living human beings to other stars are formidable. To travel many light years within the human lifespan would need enormous amounts of energy, more that the annual world production. In reference [48] the currently visible possibilities are discussed; the difficulties appear insurmountable, the most obvious one being the enormous cost of energy.

Smaller stars burn out more slowly, so planets of such stars have a habitable zone of longer lifetime. It is plausible then - again if interstellar space travel were feasible-human kind would look for a suitable planet orbiting a small star to extend its duration of existence by many tens of billions of years. In this case, work on a data storage system for a duration that long might make sense, but the design and manufacturing of such a system would be the problem of the people who colonize such a planet.

Two extremes of a data storage system can be considered: a small one, which is inexpensive enough to fabricate in vast numbers (millions or billions of copies) and a big expensive one. Copies of the small system could be dispersed randomly over the earth. Having enough of them, they could be found regularly in good condition over a long time. The disadvantage of such a system is that it can only contain comparatively little information. To give an example, on a $1 \mathrm{~cm}^{2}$ coin about $1 \mathrm{~GB}$ of data could possibly be stored with current technology. (The systems we have now-magnetic recording, RAM - are designed to be reliable for 10 years only.)

The other extreme would be a large system, of which only a few copies - two or three-could be made because of the high costs. Such systems must be located at sites which are safe from great geological changes. Furthermore the systems should be protected against vandalism; therefore it must not be easy to either find, or to access. If it were difficult to find, hints about its existence should be readily available. Vandalism therefore seems to be a serious threat. Given the geological activity of Earth it seems to be debatable whether a placement on our planet is a good option, simply because a relatively small chance of the destruction of the system is not acceptable.

Placing the system on the moon instead of on earth has, so it seems, its merits. If the-perhaps optimistic - estimate of a $2 \%$ chance of it being destroyed by a small impact, then three copies a few hundred kilometers apart would have a one in a hundred thousand chance of destruction. Therefore, 
one day the system would be hit by an asteroid or comet, making neither the earth nor the moon a good option to store information to last 500 million years.

Figure 4. Lifetime distribution of families. Data from [43].

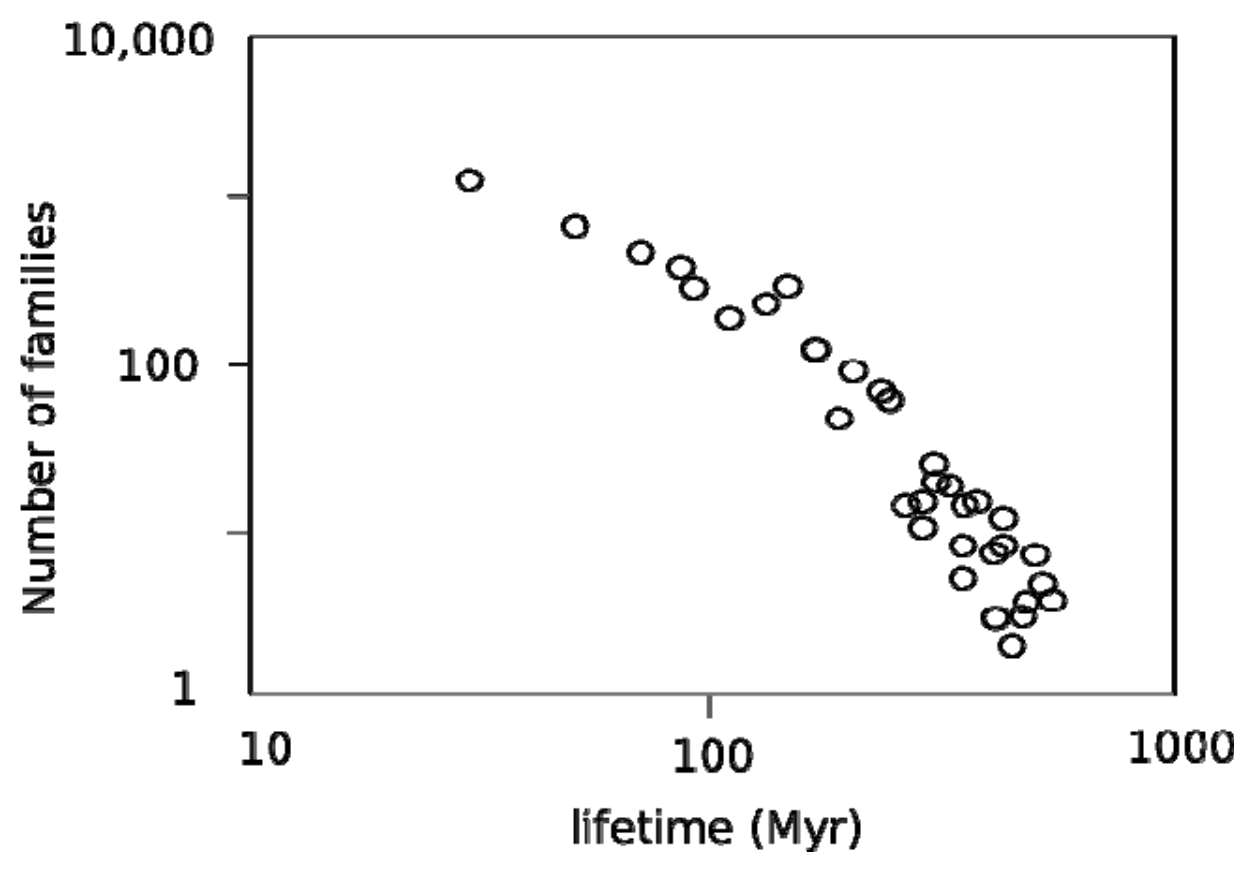

\section{Acknowledgement}

This paper was inspired by many discussions with Andreas Manz (FRIAS, University of Freiburg) and with Leon Abelmann (MESA+, University of Twente). Andreas Manz coined the idea of a "Human Document Project" to store information on human kind for one million years. This paper is based on a lecture the author has given at a workshop on the Human Document Project [49].

\section{References}

1. Damour, T.; Dyson, F. The Oklo bound on the time variation of the fine-structure constant revisited. Nucl. Phys. 1996, B480, 37-54.

2. Adkins, L. Empires of the Plain: Henry Rawlinson and the Lost Languages of Babylon; St. Martin's Press: New York, NY, USA, 2003.

3. Baty, J.W.; Sinnott, M.L. The kinetics of the spontaneous, proton- and AlIII-catalysed hydrolysis of 1,5-anhydrocellobiitol-Models for cellulose depolymerization in paper aging and alkaline pulping, and a benchmark for cellulase efficiency. Can. J. Chem. 2005, 83, 1516-1524.

4. Heminger, A.R.; Robertson, S.B. Digital Rosetta stone: A conceptual model for maintaining long-term access to digital documents. J. Management Inf. Sys. 2005, 21, 11-35.

5. Coles, P. The state of the Universe; Nature 2005, 433, 248-256.

6. Weinberg, S. The First Three Minutes; Basic Books: New York, NY, USA, 1993. 
7. Jarosik, N.; Bennett, C.L.; Dunkley, J.; Gold, B.; Greason, M.R.; Halpern, M.; Hill, R.S.; Hinshaw, G.; Kogut, A.; Komatsu, E.; Larson, D.; Limon, M.; Meyer, S.S.; Nolta, M.R.; Odegard, N.; Page, L.; Smith, K.M.; Spergel, D.N.; Tucker, G.S.; Weiland, J.L.; Wollack, E.; Wright, E.L. Seven-year Wilkinson Microwave Anisotropy Probe (WMAP1) observations: Sky maps, systematic errors, and basic results. Available online: http:/lambda.gsfc.nasa.gov/ product/map/dr4/pub_papers/sevenyear/basic_results/wmap_7yr_basic_results.pdf (accessed on 28 July 2010).

8. Lineweaver, C.H.; Fenner, Y.; Gibson, B.K. The Galactic habitable zone and the age distribution of complex life in the Milky Way. Science 2004, 303, 59-62.

9. Krauss, L.M.; Starkman, G.D. The fate of life in the universe. Sci. Am. 1999, 281, 58-65.

10. Dyson, F.J. Time without end-Physics and biology in an open universe. Rev. Mod. Phys. 1979, 51, 447-460.

11. Krauss, L.M.; Starkman, G.D. Life, the universe, and nothing: Life and death in an ever-expanding universe. Astrophys. J. 2000, 531, 22-30.

12. Ostriker, J.P.; Steinhardt, P.J. The quintessential universe. Sci. Am. 2001, 284, 46-53.

13. Cox, T.J.; Loeb, A. The collision between the Milky Way and Andromeda. Monthly Notices Roy. Astr. Soc. 2008, 386, 461-474.

14. Mojzsis, S.J.; Arrhenius, G.; McKeegan, K.D.; Harrison, T.M.; Nutman, A.P.; Friends, C.R.L. Evidence for life on earth before 3.8 billion years ago. Nature 1996, 384, 55-59.

15. Lovelock, J.E.; Whitfield, M. Life span of the biosphere. Nature 1982, 296, 561-563.

16. Carroll, B.W.; Ostlie, D.A. An Introduction to Modern Astrophysics; Pearson Addison Wesley: San Francisco, CA, USA, 2007.

17. Lovelock, J.E.; Margulis, L. Atmospheric homeostasis by and for biosphere-Gaya hypothesis. Tellus 1974, 26, 2-10.

18. Ward, P.D.; Brownlee, D. Rare Earth-Why Complex Life in Uncommon in the Universe; Copernicus Books: New York, NY, USA, 2000.

19. Ward, P.D.; Brownlee, D. The Life and Death of Planet Earth; Piatkus: London, UK, 2007.

20. Davis, M.; Hut, P.; Muller, R.A. Extinction of species by periodic comet showers. Nature 1984, 308, 715-717.

21. Zimmer, C. Evolution - the Triumph of an Idea; HarperCollins: New York, NY, USA, 2001.

22. Portegies Zwart, S.F. The long lost siblings of the sun. Sci. Am. 2009, 301, 40-47.

23. Briceño, C.; Vivas, A.K.; Calvet, N.; Hartmann, L.; Pacheco, R.; Herrera, D.; Romero, L.; Berlind, P.; Sánchez, G.; Snyder, J.A.; Andrews, P. The CIDA-QUEST large-scale survey of Orion OB1: Evidence for rapid disk dissipation in a dispersed stellar population. Science 2001, 291, 93-96.

24. Benitez, N.; Maiz-Apellaniz, J.; Canelles, M. Evidence for nearby supernova explosions. Phys. Rev. Lett. 2002, 88, 081101.

25. Site of the Planetary and Space Science Center. University of Brunswick: Fredericton, NB, USA, 2010; Available online: http://www.unb.ca/passc/ImpactDatabase/ (accessed on 1 July 2010).

26. Palme, H. The giant impact formation of the moon. Science 2004, 304, 977-979.

27. Hut, P.; Alvarez, W.; Elder, W.P.; Hansen, T.; Kauffman, E.G.; Keller, G.; Shoemaker, E.M.; Weissman, P.R. Comet showers as a cause of mass extinctions. Nature 1987, 329, 118-126. 
28. Spray, J.G.; Kelley, S.P.; Rowley, D.B. Evidence for late Triassic multiple impact event on Earth. Nature 1998, 392, 171-173.

29. Toon, O.B.; Zahnle, K.; Turco, R.P.; Covey, C. Environmental perturbations caused by the impacts of asteroids and comets. Rev. Geophys. 1997, 35, 41-78.

30. Benton, M. When Life Nearly Died: The Greatest Mass Extinction of All Time; Thames \& Hudson: New York, NY, USA, 2003.

31. Zolotukhin, V.V.; Al'Mukhamedov, A.I. Traps of the Siberian platform. In Continental Flood Basalts; Macdougall, J.D., Ed.; Kluwer Academic Publishing: Amsterdam, The Netherlands, 1988; pp. 273-310.

32. Gibbons, A. Pleistocene population explosions. Science 1993, 262, 27-28.

33. Rampino, M.R.; Self, S. Bottleneck in human evolution and the Toba eruption. Science 1993, 262, 1955-1955.

34. Oppenheimer, C. Limited global change due to the largest known Quarternary eruption, Toba approximate 74 kyr BP? Quaternary Sci. Rev. 2002, 21, 593-1609.

35. Ward, P.D. Impact from the deep. Sci. Am. 2006, 295, 64-71.

36. Le Feuvre, M.; Wieczorek, M.A. Nonuniform cratering of the terrestial planets. Icarus 2008, 197, 291-306.

37. Chapman, C.R.; Morrison, D. Impacts on the earth by asterioids and comets-Assessing the hazard. Nature 1994, 367, 33-40.

38. Wiesel, W. The Meteorite Flux at the Lunar Surface. Icarus 1971, 15, 373-383.

39. De Pater, I.; Lissauer, J.J. Planetary Sciences; Cambridge University Press: Cambridge, UK, 2001.

40. McCallum, M.L. Amphibian decline or extinction? Current declines dwarf background extinction rate. J. Herpetol. 2007, 41, 483-491.

41. May, R.M.; Lawton, J.H.; Stork, N.E. Assessing extinction rates. In Extinction Rates; Lawton, J.H., May, R.M., Eds.; Oxford University Press: Oxford, UK, 1995; pp. 1-24.

42. Wilson, D.E.; Reeder, D.A.M. Mammal Species of the World; John Hopkins University Press: Baltimore, MD, USA, 2005.

43. Yohsuke, M.; Shimada, T.; Ito, N. A simple model for skewed species-lifetime distributions. New J. Phys. 2010, 12, 063021.

44. Tattersall, I.; Schwartz, J. Extinct Humans; Westview Press: New York, NY, USA, 2000.

45. Reed, D.L.; Smith, V.S.; Hammond, S.L.; Rogers, A.R.; Clayton, D.H. Genetic analysis of lice supports direct contact between modern and archaic humans. PLoS Biol. 2004, 2, e340.

46. Green, R.E.; Krause, J.; Briggs, A.W.; Maricic, T.; Stenzel, U.; Kircher, M.; Patterson, N.; Li, H.; Zhai, W.; Hsi-Yang Fritz, M.; Hansen, N.F.; Durand, E.Y.; Malaspinas, A.-S.; Jensen, J.D.; Marques-Bonet, T.; Alkan, C.; Prüfer, K.; Meyer, M.; Burbano, H.A.; Good, J.M.; Schultz, R.; Aximu-Petri, A.; Butthof, A.; Höber, B.; Höffner, B.; Siegemund, M.; Weihmann, A.; Nusbaum, C.; Lander, E.S.; Russ, C.; Novod, N.; Affourtit, J.; Egholm, M.; Verna, C.; Rudan, P.; Brajkovic, D.; Kucan, Ž.; Gušic, I.; Doronichev, V.B.; Golovanova, L.V.; Lalueza-Fox, C.; de la Rasilla, M.; Fortea, J.; Rosas, A.; Schmitz, R.W.; Johnson, P.L.F.; Eichler, E.E.; Falush, D.; Birney, E.; Mullikin, J.C.; Slatkin, M.; Nielsen, R.; Kelso, J.; Lachmann, M.; Reich, D.; Pääbo, S. A draft sequence of the Neandertal Genome. Science 2010, 328, 710-722. 
47. Schmidt, H.; Borchardt, G.; Rudolphi, M. ; Maumann, H.; Bruns, M. ; Weber, S. ; Scherrer, H. Nitrogen self-diffusion in polycrystalline Si3N4 films: Isotope heterostructures vs. gas-exchange. Defect and Diffusion Forum 2005, 237-240, 512-517; doi: 10.4028/www.scientific.net/DDF.237240.512.

48. Beardsley, T. The way to go to space. Sci. Am. 1999, 280, 58-76.

49. Human Document Project; Workshop, Saarbrücken, Germany, 1-2 July 2010.

(C) 2011 by the authors; licensee MDPI, Basel, Switzerland. This article is an open access article distributed under the terms and conditions of the Creative Commons Attribution license (http://creativecommons.org/licenses/by/3.0/). 